\title{
À PROPOS DU « PHÉNOMÈNE » ET DE LA "PHÉNOMÈNALISATION » CHEZ MARC RICHIR
}

\author{
ALEXANDER SCHNELL
}

\begin{abstract}
This article is dedicated to Richir's basic understanding of the phenomenon and the phenomenalisation. It is divided into three parts. The first part deals with Richir's concept of the phenomenon in general and the reference to the concept of the "transcendental" in particular. The second part is devoted to the concept of "phenomenalisation" in the "Notes sur la phénoménalisation" (1969-70), which has remained unpublished to this day. The focus is on the decoupling of "phenomenality" and "eideticity", the explanation of the "circle of foresight", the "ontic-ontological circle" and the question of the relationship between "phenomenalisation" and "writing". The third part is interested in the connection between "phenomenalisation" and "architectonics" in the late Richir. In it, "architectonics" is emphasized as a specific mode of phenomenalization.
\end{abstract}

L'objectif de cette contribution est d'aborder un point méthodologique primordial dans l'œuvre de Richir qui concerne le sens du phénomène et - point non moins important dont relève la phénoménalisation - la manière d'y accéder. Ces deux concepts ne concernent pas cette œuvre de façon seulement interne, mais renvoient d'une façon plus générale à la problématique fondamentale du rapport entre apparaître et être en phénoménologie.

Qu'est-ce qui caractérise en propre la phénoménologie ? Question sans doute pas illégitime puisque tous ceux qui la soulèvent semblent y reconnaître un signe distinctif. Or, l'acception richirienne de la phénoménologie n'est peut-être pas celle qui permette le mieux d'identifier le caractère commun de toutes les élaborations phénoménologiques actuelles (en revanche, elle est exemplaire pour la phénoménologie comprise comme philosophie transcendantale - et c'est à ce titre que https://doi.org/10.14712/24646504.2020.13

(C) 2020 The Author. This is an open-access article distributed under the terms of the Creative Commons Attribution License (http://creativecommons.org/licenses/by/4.0). 
le projet richirien doit au plus haut point attirer notre attention). Il n'empêche que Richir lui-même considérait sa propre compréhension de la phénoménologie comme la seule valable et acceptable. En témoigne la note éditoriale des Annales de Phénoménologie qui s'ouvre précisément par la question de savoir " en quel sens y sera pris le terme de phénoménologie ». Dans ce qui suit, il sera question du phénomène et de la phénoménalisation selon trois perspectives différentes. Nous proposerons dans un premier moment une analyse d'un assez long extrait de cette note qui débouchera sur une élimination de l'être en phénoménologie (le reléguant au statut de simulacre); nous ferons ensuite des remarques sur la phénoménalisation chez le tout jeune Richir qui ouvre un mode du phénoménaliser différent de toute forme d'expression; et enfin, nous nous interrogerons sur la fonction de l'architectonique dans la méthodologie richirienne tardive qui permet de promouvoir encore un autre sens - non moins original et inédit - du phénoménaliser.

\section{Le sens de la phénoménologie d'après Marc Richir}

En 2002, Richir affirme avec force dans sa note éditoriale susmentionnée :

lors que le discours phénoménologique ne peut avoir de sens précis que s'il donne très précisément à entendre de quoi (de quel problème ou question), chaque fois, il parle, et que c'est ce "quoi " (la Sache selbst, la "chose même ") qui doit par là être attestable (directement ou indirectement) dans l'effectuation (au sens mathématique) de l'opération qui permet d'y accéder, le caractère de la spéculation qui a perdu sa rigueur classique (sa «logique " propre, bien au-delà de la logique) est que n'importe quoi peut y être à peu près dit de n'importe quoi, à condition que la « construction » spéculative ait plus ou moins bien l'air de tenir, souvent (mais pas toujours) dans les enchaînements de pseudo-concepts (ineffectuables) relevant plus du bricolage ou de l'idéologie que de la "logique " méthodiquement déployée d’une élaboration qui se donne ses règles et ses angles d'attaque des problèmes. [...]

L'œuvre de Husserl que, depuis la publication des Husserliana, on ne peut plus lire aujourd'hui comme il y a cinquante ans, est un immense chantier où il n'est pas une seule question qui [ne] soit un problème à reprendre, à réélaborer, à redéfinir, éventuellement, selon d'autres " axes de coordonnées ", cela à la fois par la mise [au] jour des contextes historiques concrets où la pensée husserlienne s'est déployée, et par des recherches autrement orientées portant sur les " choses mêmes » que Husserl avait touchées, ou qui n’y étaient encore que secrètement «impliquées ». [...]

Cependant, pour faire vivre ou revivre cet esprit, il s'agit [...] de lever bien des obstacles, de comprendre le sens husserlien du phénomène (qui n'est ni apparition, ni 
apparaissant, ni le jeu inapparent des deux), et le sens husserlien du transcendantal (qui n'est pas réductible à l'ordre de la condition de possibilité a priori en son acception kantienne, mais relève d'un a priori fungierend, en fonction, qu'il s'agit de dévoiler dans ses complexités par l'épochè et la réduction, puis par l'analyse en zigzag). Bien des ressources demeurent insoupçonnées pour l'analyse des structures intentionnelles complexes (les phénomènes husserliens), opérantes même depuis leurs potentialités multiples, c'està-dire même hors du présent, voire de la présence. [...]

Par là déjà, il apparaît que le champ phénoménologique husserlien - quoi qu'on puisse y trouver à redire par ailleurs - est bien plus vaste que ce qui est réductible à l'un ou l'autre projet ontologisant. La question du phénomène en son sens husserlien, et donc en son sens révolutionnaire, proprement phénoménologique, n'a que très secondairement et très localement à voir avec la question de l'être.

Ces réflexions s'inscrivent dans un contexte plus polémique (encore) que ne le laissent entendre ces lignes; elles ont d'ailleurs valu à l'ancienne ${ }^{1}$ Association pour la promotion de la phénoménologie, de la part de certaines personnalités qui devaient faire partie de son noyau dur - comme Rudolf Bernet -, un refus de participer à cette aventure. Mais laissons de côté ces détails anecdotiques et penchons-nous sur ce qui est dit. À notre avis, il faut ici mettre en avant les points suivants :

1) La Sache, le quoi dont parle le discours phénoménologique, n'est rien de directement donné, mais, premièrement, elle se déploie dans une élaboration qui, d'une part, doit être effectuée et, d'autre part, possède une logique propre qui fournit ses règles ainsi que les angles d'attaque à partir desquelles elle peut être analysée ; et, deuxièmement, ce même discours phénoménologique exige que l'on dévoile ce qui est "impliqué », "voilé ", " hors du présent, voire de la présence ». Donc la Sache s'atteste dans une effectuation qui n' est nullement simplement là, mais toujours à faire, et qui doit être accompagnée dans son se-faire propre (le « sens se faisant "!) ; et, dans cette élaboration effectuée - et sans cesse de nouveau à effectuer - est porté au jour non pas ce qui est institué, mais le phénoménologique. Qu'est-ce à dire?

2) Il y va d'un nouveau sens du phénomène et du transcendantal, qui ne sont autres, pour Richir, que ceux-là mêmes en vigueur chez Husserl (pour peu, toutefois, qu'il soit bien compris). Or, si le « sens husserlien du phénomène » n'est, d'abord, citons à nouveau, «ni apparition, ni apparaissant, ni le jeu inapparent des deux [c'est nous qui soulignons] », alors se pose la question de savoir lequel

1 Depuis 2019, cette association - qui édite les Annales de Phénoménologie-Nouvelle série ainsi que les Mémoires des Annales de Phénoménologie - porte le titre « Association Internationale de Phénoménologie (A.I.P.) ». 
il est. Réponse : il n'est pas le « jeu inapparent » de l'apparition, ni non plus celui de l'apparaissant - mais das Unscheinbare comme tel, die Unscheinbarkeit comme telle. Cela veut dire : non pas le jeu inapparent d'un quelconque apparent, mais précisément le "'jeu' inapparent " lui-même! Encore faut-il bien s'entendre sur le statut de l'inapparent. Il ne s'agit pas de quelque chose qui serait déplacé du champ de la présence et de la manifestation vers une sphère invisible, et qui devrait d'abord être tiré vers la lumière. Il ne s'agit d'aucune objectité, mais d'un Fungieren, d' " opérations " " en fonction ", dont le processus de " dévoilement » exige l'épochè, la réduction et l'analyse en zigzag ainsi que des " concepts opératoires » dont le statut doit être spécifié.

L'épochè et la réduction: Richir est de part en part - et c'est bien connu - un penseur du mouvement. Cela concerne dans une large mesure aussi sa conception de l'épochè et de la réduction, justement. Il pense la différence entre ces deux termes de façon originale, tout en suivant une perspective d'abord ouverte par Patočka ${ }^{2}$. L'idée fondamentale du phénoménologue tchèque consistait, on le sait, à effectuer une radicalisation de l'épochè préparant ce qu'il a appelé une "phénoménologie asubjective ». Pour Richir, l'épochè et la réduction sont liées de façon encore plus étroite l'une à l'autre, la réduction fixant en quelque sorte ce que l'épochè avait d'abord défait. L'épochè n'est pas, négativement, une simple mise hors circuit ou une mise en suspens, mais, positivement, une ouverture. L'épochè ouvre à la dimension fluide du sens (par opposition à l'apparente fixité des objectivités « real»). Et la réduction creuse l'en-deçà par rapport à l'au-delà que constitue cette ouverture de la fluidité (et que Richir a appelé, dans ses dernières élaborations, une « hyperbole »). La réduction fait apparaître une certaine positivité là où tout s'éparpille et s'émiette à l'infini. Et il faut bien insister sur ce rapport étroit, médiatisé à travers l'idée de la "positivité »: l'épochè transcende la positivité (au sens où Heidegger avait entendu le terme de transzendieren dans l'important volume 26 de la Gesamtausgabe) afin de faire apparaître ce qui la fait vibrer, clignoter ou vivre ; et ensuite, la réduction assume ou prend sur elle la positivité (qui, bien entendu, n'est pas celle de l'objectivité « real» mais du phénomène lui-même), pour mettre en évidence, précisément, la sphère de l'en-deçà du phénomène. Nous ajouterons encore une dernière chose à propos de ce couple de concepts méthodologiques essentiels : Richir a au fond peu réfléchi (sur) sa propre méthode. Ce qui l'explique, c'est que sa méthode s'inscrit dans le mouvement même de sa pensée. L'une des conséquences en est que cette remarque méthodologique à propos de l'épochè

2 Voir Patočka Jan, «Epochè et réduction », in: Patočka, Jan, Qu'est-ce que la phénoménologie?, trad. par E. Abrams, coll. « Krisis », Grenoble, Millon, 1988, pp. 249-261 ; Patočka J, Qu'est-ce que la phénoménologie?, op. cit., pp. 263-302. 
et de la réduction est motivée par une idée que l'on trouve aussi ailleurs. Nous ne nous référerons à ce propos qu' au moment du sublime qui revient partout dans les derniers écrits de Richir. Peut-être que l'interrelation entre l'épochè et la réduction - isolée de façon thématique et quelque peu artificielle dans cette réflexion méthodologique - fait d'une certaine façon écho (mais dans une configuration inversée !) à la systole et à la diastole du moment du sublime. Peut-être l'ouverture fixante et fixée a-t-elle besoin d'un relâchement qui fait suite à une condensation et concentration proto-ontologique, affective, mais toujours virtuelle. Quoi qu'il en soit, ce peut-être renvoie sans doute à un rapport spécifique entre la méthode et l'architectonique, auquel nous reviendrons dans un instant.

L'analyse en zigzag : entre quelles entités cette fameuse analyse en zigzag s'effectue-t-elle? Certes déjà entre les objectivités mêmes qui, tout en étant mises entre parenthèses eu égard à leur être en soi, n'en servent pas moins, avec leur typique et leurs motivations, de fils conducteurs à l'analyse phénoménologique. Mais l'essentiel n'est pas là. Il y a aussi un zigzag plus profond, plus enfoui comme Richir aimait à dire, entre la dimension apparaissante en régime d'épochè et la dimension inapparente qui constitue précisément le phénomène (ou ce que Richir appelle le phénoménologique). Ou encore, tout simplement, entre la fluidité phénoménale, d'un côté, et la positivité pré-phénoménale, pré-immanente, de l'autre - une positivité qui est marquée par le sceau de la négativité qui avait occupé Richir dans De la négativité en phénoménologie (2014) et qui permet de comprendre pourquoi, aux yeux de Richir, l'attitude phénoménologique rigoureusement maintenue rend impossible un retour à la naïveté de l'attitude naturelle.

3) Le troisième point - qui est peut-être le plus subtil, voire le plus difficile - concerne le rapport entre la phénoménologie (richirienne) et l'ontologie. Comme la note éditoriale rappelée plus haut l'affirmait sans ambages, Richir considère que le champ phénoménologique s'étend largement au-delà de celui « de l'un ou l'autre projet ontologisant ». Par "l'un ou l'autre », il faut d'abord entendre, cela va de soi, le projet heideggérien et peut-être, mais dans tous les cas autrement, celui de Levinas. L'arrière-fond a ici toutefois une portée systématique qui va bien au-delà d'un débat polémique avec les auteurs de Sein und Zeit et d'Autrement qu'être ou au-delà de l'essence. C'est qu'il s'agit pour Richir de régler ses comptes avec la tradition philosophique reposant sur l'argument ontologique. Au centre de ce règlement de compte est la notion du simulacre.

Concernant la positivité en régime phénoménologique, nous disions à l'instant qu'elle était marquée par le sceau d'une négativité. Or, traditionnellement, et c'est évidemment à la tradition (notamment platonicienne) que Richir s'adresse ici, l'être qui est entaché de négativité, de non-être, s'appelle « l'apparence (Schein)». 
Les phénoménologues, avant tout ceux de la deuxième génération de phénoménologues (de langue française), ont souvent joué sur la polysémie de l'apparence, du paraître, de l'apparoir, entre l'apparition et l'apparaître, d'un côté, et la simple apparence ou l'illusion, de l'autre. C'est précisément à l'endroit de cette équivoque, de cette ambiguité, que se place aussi Richir. Mais il va plus loin, il en dégage un sens phénoménologique profond. L'essentielle difficulté qui caractérise la positivité en régime phénoménologique, c'est le fait qu'aucune réalité pré-donnée, aucune objectivité préétablie, aucun être en soi ne saurait servir d'échelle à l'aune de laquelle se mesurerait la Stimmigkeit (que l'on pourrait traduire par adéquation, si l'on avait le droit de se référer au cadre traditionnel de la vérité-correspondance) de la positivité phénoménologique. Du coup, celle-ci est en permanence menacée d'être illusoire. Dit autrement, la positivité donne partout lieu à des apparences de la positivité. L'exploitation radicale de cette idée a été tentée au moins deux fois dans l'œuvre richirienne : dans ses Recherches phénoménologiques et, plus puissamment encore, à partir de Phénoménologie en esquisses. Nouvelles fondations, où la phantasía (dans ses rapports subtils et intimes aux transpositions imaginatives) se substitue au lexique de l'apparence, de l'illusion, du simulacre.

Les conséquences ontologiques de ce qui vient d'être affirmé permettent de comprendre la critique richirienne de l'argument ontologique de la tradition métaphysique : Richir ne traque pas simplement cet argument chez Anselme, Descartes ou Leibniz afin d'en dénoncer l'influence sur la tradition philosophique moderne, mais il affirme tout bonnement que toute assomption d'un être ou d'êtres relève $d u$ simulacre! Il n'y a pas d'un côté l'être et de l'autre côté l'apparence; aussi le passage de la phénoménalité comme rien que phénoménalité à un être transcendant, voire même corrélatif, est-il, à ses yeux, totalement inacceptable. Comprendre Richir implique de comprendre sa conception de la réalité, et comprendre cette dernière requiert de généraliser, en toute radicalité, la mise hors circuit - une fois pour toutes et à jamais - de tout argument ontologique. Aussi le phénomène et le phénoménologique ne relèvent-ils pas simplement de l'inapparence, mais également, et en toute rigueur, du non-être (car l'être est toujours caractérisé, pour Richir, d'une certaine « fixité »).

\section{2. À propos de la phénoménalisation chez le jeune Richir}

Or, ce dernier point apparaissait très tôt déjà, chez Richir, dans ses notes (encore inédites jusqu'à ce jour) sur la phénoménalisation. Voyons à présent quelle autre perspective celles-ci ouvrent eu égard au sens et au statut du phénoménaliser. 
Que veut dire phénoménalisation ? On peut mettre en évidence différents motifs qui la déterminent fondamentalement et qui trouvent leur origine dans les premières lectures richiriennes de Husserl et de Heidegger ${ }^{3}$.

Concernant Husserl, Richir affirme, dans Le problème de la phénoménalisation, que celle-ci doit être considérée comme étant en lien avec l'idée d'horizon défini 1) comme idée au sens kantien et 2) comme forme vide (ni présente, ni absente ; et infinie, c'est-à-dire n'étant pas l'objet d'une intuition finie), donc comme forme vide recueillant les apparitions en vue des apparaissants.

D’après Heidegger, en revanche, la phénoménalisation pense le mouvement du cercle de la précompréhension (au sens de la vision [théôria] platonicienne). Ce mouvement consiste (au niveau de l'ustensilité, donc des choses de la vie quotidienne avant leur objectivation théorique et gnoséologique) à pré-saisir dans la voyance (intuition) la façon dont « l'étant vient à être étant, par quoi l'étant vient à paraître, i.e. à être révélé (découvert) dans son être. Ainsi, Heidegger parvient-il à thématiser le cercle de la vision, en ce que le dessin de ce cercle (son inscription) est ce qui est constitutif de l'être-là. La compréhension [...] de ce cercle ouvre alors, dans le mouvement de le dé-crire (i.e. dé(sins)crire) la question du sens de l'être, en tant que ce mouvement 'est' le mouvement par lequel l'être dessine en son cercle le site en quoi viendra à paraître l'étant $»^{4}$.

Richir se propose alors de s'attaquer à la tâche suivante (annoncée dans cette même note du 12 janvier 1969) :

En d'autres termes, Heidegger se meut toujours déjà dans le cercle. Ce qui reste impensé, c'est le TRACEMENT du cercle, c'est-à-dire le mouvement qui phénoménalise, mouvement aveugle non finalisé (même pas par l'ustensilité) par quoi le finalisé (l'habitation mondaine) vient à être.

Ceci pose la difficile question d'un avant et d'un après. En tant que cet « avant » est " avant la finalité », il est sans temps et sans espace, il est non-sens, zéro de sens, rien informel. Le problème est donc celui de l'instauration de la finalité, c'est-à-dire de $l^{\prime}$ « invention » de l'outil ${ }^{5}$.

Richir entrevoit alors deux directions possibles pour penser la phénoménalisation - celle de Max Loreau, et celle de Derrida. Pour le premier, il faut parvenir à penser " hors du cercle». Deux assises sont ici envisageables : l'homme-singe qui a inventé l'outil et l'artiste (créant des formes nouvelles), tous les deux ne possédant

3 Cf. le texte du 12 janvier 1969 dont le titre est «Le problème de la phénoménalisation ».

4 Idem. On remarque ici le rôle prépondérant de l'écriture (motif éminemment derridien).

5 Idem. 
rien. Derrida vise, quant à lui, «la pensée de l'écriture ou plutôt la pensée comme écriture, comme jeu du différer qui est comme tel l'instauration de la trace, et de là, de la présence. Donc, le jeu du différer comme opération de phénoménalisation ${ }^{6}$.

Mais ici Loreau intervient à nouveau : Comment passer en effet du jeu chaotique (i.e. de l'effervescence non finalisée du corps, du règne absolu de la maladresse et du balbutiement-babillage) au jeu du différer, c'est-à-dire du chaos d'éléments au jeu de la mise en réserve? Autrement dit, comment penser la première trace? Comment le hasard se (trans)forme-t-il en non-hasard, en instauration du circuit du sens par lequel le geste délié se lie en geste finalisé dans et par l'outil ? Richir voit surgir ici un problème fondamental :

Celui de l'avant et de l'après, celui de la "première " trace, ce qui pose le problème de la genèse, qui est tout à fait négligé par Heidegger (celui-ci reste [pour Richir] un penseur de l'Histoire, sous le chef de l'historialité de l'Être qui apparait comme l'histoire (le destin : Geschichte - Geschick) des possibles, le déploiement et le développement herméneutique du cercle) et celui de la temporalisation.

C'est sans doute encore penser métaphysiquement que de chercher une "première trace ", c'est-à-dire faire de la trace une présence. Il ne s'agit pourtant moins de cela que d'une recherche des " conditions de possibilité » de la trace, c'est-à-dire, d'abord, une recherche portant sur la logique de la trace, celle-ci se mouvant dans un cercle de style heideggérien, puisque l'établissement de la logique de la trace présuppose la trace. Ce qu'il faut penser, c'est le devenir-trace de la trace, la mise en économie du jeu.

À ce point de vue, la théorie de la réduction phénoménologique contient sans doute " en creux ", en un " point » de son articulation, ce devenir-trace quand Husserl passe, d'un geste qui reste tout à fait impensé, du jeu du monde (le chaos d'esquisses) à l'absolu transcendantal. Il faudrait radicaliser cette "fiction ", éliminer le présupposé d'une conscience transcendantale, donc creuser, davantage encore, ce qui est entrepris dans mon mémoire.

Peut-être faudrait-il dégager, dans le " creux » des recherches génétiques de Husserl, une problématique radicale de la genèse qui permettrait d'accéder à une pensée du devenir-trace de la trace, de la transgression par laquelle le hasard devient sens (cf. Méditations cartésiennes : "c'est l'expérience, muette encore, qu'il s'agit d'amener à l'expression pure de son sens $»$ ).

Le problème de l' "invention de l'outil ", de l'instauration d'une finalité (dans le geste discipliné par, pour, et dans l'outil) passe donc par le problème de la phénoménalisation et de la temporalisation. C'est aussi le problème de l' " origine » de l'écriture.

En effet, on peut ici esquisser la "régression » vers les « origines » que tente Derrida. Lécriture elle aussi fut pensée comme un instrument (et encore par Heidegger dans Sein und Zeit, cf. p.168, et plus généralement, les $\$ \$ 34-35$ ). La question de la naissance

6 Idem. 
de l'outil est donc « analogue » à la question de la naissance de l'écriture. Il faut donc déconstruire le côté "instrument " de l'outil, y déceler "l'instauration " de la trace, «l'archi-mouvement » de l'archi-écriture. L'outil est «simultanément » instauration de la trace et d'une finalité.

Peut-on penser la trace sans l'inscrire dans un cadre de finalité ? Tout est là7.

Or, Loreau prend précisément pour point de départ la pensée non finalisée qui se finalise en se traçant dans l'art (ce qui exige de " déconstruire la pensée heideggérienne de l'art, voir en quoi son point de départ dans l'ustensilité lui fait 'manquer' le délire dubuffétien d'inscription graphique »). Mais il reste ici pour Richir encore trop proche de Platon.

Les réflexions de Richir vont alors dans le sens d'une réduction radicalisée, capable de déraciner la Weltapperzeption, afin d'accéder à un type de phénoménalisation en deçà de toute métaphysique ou onto-théologie.

Une autre idée essentielle, concernant la phénoménalisation - peut-être la plus importante -, se trouve dans l'Exposé succinct de l'état actuel de nos recherches ( $d u$ 20 au 24 janvier 1969). Dans ce texte de tout premier ordre, Richir revient sur son mémoire de Licence de 1968 (intitulé La fondation de la phénoménologie transcendantale (1887-1913), dont un exemplaire se trouve à la bibliothèque des Archives Husserl de Leuven) et identifie ce qu'il appelle le problème phénoménologique (que l'on trouve autant dans l'introduction au volume II des Recherches Logiques - texte essentiel pour Richir - que, formellement, dans la Phénoménologie de l'esprit de $\mathrm{Hegel}^{8}$ ). La problématique générale est la suivante : il s'agit, pour Husserl, de clarifier les concepts logiques afin de pouvoir livrer, sur cette base, un fondement apodictique à la logique. Or, un tel fondement ne saurait être trouvé que dans le Bewusstseinserlebnis (vécu de conscience) dans lequel le concept logique est visé. Est du coup requise une description pure de ce vécu. Citons alors le passage essentiel dans lequel le problème phénoménologique évoqué à l'instant trouve sa formulation la plus expresse (et qui anticipe toute l'œuvre suivante de Richir au moins jusqu'aux Méditations phénoménologiques) :

\footnotetext{
Idem.

8 Pour la réception richirienne de Hegel, la lecture de Max Loreau de la Phénoménologie de l'esprit fut essentielle ; voir Loreau Max, «Lecture de l'Introduction à la Phénoménologie de l'Esprit de Hegel », in Textures, 69/5, pp. 3-34 (cet article a été reproduit dans En quête d'un autre commencement, éditions Lebeer Hossmann, Bruxelles, 1987, sous le titre : «L'introduction à la phénoménologie de l'esprit de Hegel », pp. 11-42); Loreau Max, « Hegel et le corps récalcitrant (Lecture de la 'Certitude Sensible', chapitre ler de la Phénoménologie de l'Esprit de Hegel) », in Textures, 70/7-8, pp. 55-102 (cet article a également été reproduit dans En quête d'un autre commencement sous le titre : « Hegel et le corps récalcitrant (La certitude sensible) », pp. 43-89).
} 
Pour que la description du vécu soit pure, c'est-à-dire pour que rien de ce qui est à expliciter ne soit présupposé dans la description [c'est là un mot d'ordre formulé explicitement dans l'introduction au volume II des Recherches logiques], il faudrait que l'intuition du vécu à décrire ne soit en rien contaminée par l'intuition préalable de l'idéalité logique visée par le vécu ; il faudrait que le regard intuitif portant sur le vécu puisse se séparer purement du regard portant sur l'idéalité, donc que le regard se dédouble de manière à ce que l'une de ses parties soit rigoureusement indépendante de l'autre. Or, c'est là une position impossible : si le regard qui porte sur le vécu ne sait plus que ce vécu est précisément le vécu en lequel l'idéalité à clarifier est visée, plus rien ne permet de voir que l'acte de pensée à décrire est l'acte de pensée de cette idéalité. Autrement dit, la corrélation entre le vécu et l'idéalité est brisée et la description phénoménologique perd sa signification qui est précisément de clarifier l'idéalité visée. $\mathrm{Ce}[\mathrm{la}]$ a pour conséquence que le vécu n'acquiert sa signification que grâce à l'idéalité à laquelle il se rattache. Cette idéalité fonctionne donc comme la lumière qui éclaire le vécu en lui donnant forme et sens, si bien que le projet phénoménologique se renverse : ce qui était pré-appréhendé comme fondement [= le vécu] paraît bien n'être que fondé [par l'idéalité]. Ce[la] montre que la description phénoménologique qui est mise en œuvre dans les Recherches [logiques] se meut dans un cercle : ce n'est que grâce à une pré-vision de l'idéalité à clarifier que la clarification est possible, ce qui est à clarifier est toujours déjà présupposé dans la clarification. Ce qui doit se trouver à la fin (l'idéalité clarifiée) est toujours déjà au début. L'idéalité joue donc le rôle d'archè et de telos. C'est parce que l'idéalité est toujours déjà pré-vue qu'elle peut finaliser la description phénoménologique du vécu. Si l'on y réfléchit, ce cercle est le cercle de la métaphysique telle qu'elle fut inaugurée par les Grecs : la vérité, comme bonne vision de l'eidos, comme exactitude du regard (orthotes, Platon, République, 515d), comme homoiôsis, accord de la connaissance et de la chose elle-même, ne peut se réaliser (c'està-dire répondre à son critère) que si l'on a déjà préalablement en vue ce qu'est cette exactitude et cet accord, donc seulement si l'eidos a déjà été pré-vue. C'est pourquoi nous baptiserons ce cercle cercle de la pré-vision. Le paradoxe est que Husserl maintient l'exigence contradictoire de décrire le vécu sans regarder l'idéalité visée dans le vécu. C'est cette situation paradoxale qui l'amena à mettre au point la théorie de la réduction phénoménologique. Sans qu'il soit possible de rentrer ici dans les détails, disons simplement que cette exigence contradictoire permet de rendre compte du caractère paradoxal de la réduction - qui, à notre connaissance, n'a jamais été repéré comme tel par les « exégètes » de Husserl - qui est la « mise hors circuit » : celle-ci n'est ni suppression - révocation cartésienne - ni néantisation, mais mise hors du circuit de la pensée, mise hors jeu. Par là, l'idéalité n'est pas rejetée dans l'absence, mais elle n'est pas non plus maintenue rivée [?] dans la présence, elle «flotte » dans une sorte d'entre-deux de la présence et de l'absence, où elle fonctionne comme un pôle vide ni présent ni absent qui donne sens au vécu. 
De la sorte, l'objectité idéale réduite (qui, après l'extension du domaine phénoménologique au-delà du domaine logique, recouvre tous les types d'objets) se donne, dès 1907 (dans Die Idee der Phänomenologie, Husserliana, B-II) comme une forme vide située à l'infini - inaccessible à l'intuition finie - indéfiniment remplissable par ce que donnent à voir les intuitions finies. C'est donc le telos d'un remplissement parfait d'une forme vide qui donne, selon Husserl, sens à l'objectivité[. ${ }^{9}$ ] Autrement dit, un étant n'est étant que dans la mesure où il s'inscrit dans le sillon pré-inscrit par l'anticipation d'un idéal (celui de la forme parfaitement remplie, donc accessible parfaitement à une intuition finie dans une présence rejetée à l'infini). Ce[la] permet de comprendre que pour Husserl, ce qui rend possible l'intuition finie, c'est l'anticipation d'horizons (de remplissement) dans le sein desquels l'étant vient à paraître. La constitution de l'étant est constitution d'une téléologie. C'est le sens de l'intentionnalité chez Husserl.

2) Notre problème

Notre problème, dont nous comptons tenter l'éclaircissement est celui-ci : Est-il possible de penser la venue au paraître de l'étant (ce que nous appellerons désormais la phénoménalisation de l'étant) sans l'inscrire dans le sillon préalablement pré-scrit par un horizon qui finalise le paraître ? La pré-scription de ce sillon est-elle nécessaire au sens ? La phénoménalisation présuppose-t-elle toujours une finalité ? La phénoménalisation est-elle nécessairement pro-duction d'un apparaître en vue de ? Est-il possible de «sortir » du cercle de la pré-vision?

Richir met ici en évidence le conflit entre le mot d'ordre d'absence de tout présupposé et l'anti-psychologisme, à savoir l'idée que le vécu de l'idéalité (qui est à clarifier) n'est pas auto-suffisant (c'est-à-dire qu'il doit savoir de quoi il est le vécu, qu'il n'obtient sa signification que grâce à ce dont il est le vécu). À lire le problème de Richir, on s'aperçoit que son idée consistant à déconnecter phénoménalité et eidéticité, mainte fois affirmée tout au long de son œuvre, est déjà présente dans ce texte de 1969.

Le projet est donc d'échapper au cercle de la pré-vision. Pour ce faire, il ne suffit pas de "balayer la tradition par la pensée pour y échapper». Aussi, Richir envisage-t-il dans un premier temps une « déconstruction de la tradition ». Celle-ci s'effectue moyennant la lecture de textes privilégiés (il se réfère ici une fois de plus à Husserl et à Heidegger).

Pourquoi cette déconstruction? Parce que les scripteurs (il est intéressant de remarquer qu'il ne dise pas auteurs) ont tendance, pour donner une cohérence à leur système, à effacer des possibles. Pour Richir, il s'agit de les " exhumer » afin d'ouvrir « la possibilité de nouvelles réponses ». Cependant, il faut éviter le danger

9 Dans le texte de Richir, il y a ici un point d'interrogation. 
de retomber dans le cercle de la pré-vision. Aussi vise-t-il une « pensée non verrouillée " (l'horizon d'une question constituant le verrou d'une pensée). Son projet consiste ainsi dans " une pensée du mouvement de déverrouillage ${ }^{10}$, c'est-à-dire une pensée de l'écriture philosophique ». Richir de récapituler : «Bref, nous serons amenés progressivement - dans le mouvement même de notre recherche - à désinscrire l'anticipation de notre horizon, à éprouver toujours plus le sens de notre question (à étudier la corrélation générale entre le but recherché et ce qui donne sens à la recherche). » La démarche qu'envisage Richir se déploie en trois temps.

1) Première thèse (en partant de Husserl) : L'anticipation de la subjectivité transcendantale - qui, en tant que présence absolue s'inscrit dans le sillon pré-scrit par l'horizon du temps - était sans doute ce qui a condamné Husserl à laisser passer ce que Richir nomme la phénoménalisation. Donc, le maintien de la subjectivité transcendantale en tant qu'elle s'inscrit dans un horizon temporel de la présence constitue l'obstacle à une pensée adéquate et appropriée de la phénoménalisation. Il s'agira ainsi de mettre à l'épreuve le concept d'horizon et de procéder à la déconstruction de la métaphysique.

2) Le caractère irréductible de l'horizon se doit d'être questionné. Cela donne deux nouvelles directions. La deuxième partie traite d'abord de Heidegger, la troisième de nouveau de Husserl.

La pensée heideggérienne de la phénoménalisation s'esquisse, pour Richir, dans la Kehre opérée dans Vom Wesen der Wahrheit (1930). ${ }^{11}$ Deuxième thèse : Heidegger échappe certes au cercle de la pré-vision, mais il reste enfermé dans un autre cercle, que Richir appelle le « cercle ontico-ontologique » et selon lequel tout étant vient se loger dans "l'horizon de l'être ». Cette pré-scription de la différence ontico-ontologique fait problème aux yeux de Richir. Heidegger effacerait la question du tracement de la différence ontico-ontologique. Il y aurait une collusion secrète entre la phénoménalisation telle que la pense Husserl et la phénoménalisation telle que la pense Heidegger, « cette phénoménalisation consistant 'formellement' en l'ouverture d'une différence entre un pôle plein présent (l'étant) et un pôle vide ni présent ni absent (l'horizon husserlien, l'être heideggérien)».

On entr'aperçoit déjà certaines réserves de Richir à l'égard de Heidegger (qui ne cesseront par la suite de s'accentuer), car il préconise de " pousser le langage ontique-métaphysique jusqu'au bout, en radicalisant la rigueur au maximum afin de le [= ce langage ontique-métaphysique de Heidegger] faire éclater ».

10 Dans le manuscrit, nous lisons «mouvement de verrouillage », mais étant donné le contexte, il s'agit manifestement d'une erreur.

11 Richir nomme également : Sur la question de l'être (1955), Identité et différence (1957) et Temps et être (1962). 
3) Enfin, il convient de lire, aux yeux de Richir, les textes de Husserl qui traitent du problème du tracement de l'horizon (articulant la question des horizons, de la téléologie et de la temporalisation). Il s'agit toujours du même problème circulaire (appliqué cette fois à la problématique du temps) : comment rendre compte de la temporalité originaire sans se donner d'avance un horizon temporel ? Les textes à mobiliser à ce dessein sont : le Manuscrit A VII (Theorie der Weltapperzeption); le Manuscrit E III (Universale Teleologie); les Manuscrits B III 3 et K III 11 (Phänomenologische Rekonstruktion = phänomenologische Archäologie) ; et enfin les Manuscrits C (dans le but de penser ensemble la phénoménalisation et la temporalisation). Ce troisième point reste à l'état de projet. Il faudrait peut-être y ajouter les Manuscrits de Bernau (dont Richir ne pouvait évidemment pas disposer à l'époque), afin de dégager une troisième thèse qui consisterait dans l'articulation entre la temporalisation originaire et une pensée de l'origine, de la genèse, qui ne part pas de la présence et qui évite ainsi la "pré-scription de l'horizon du temps».

Quelques mois plus tard, le 20 mars 1969, visiblement sous la forte influence de Derrida, Richir esquisse des réflexions intitulées Leiblichkeit du langage - Phénoménalisation. Citons, pour clore ces analyses, le passage suivant qui cherche à rendre compte de la phénoménalisation en insistant d'une autre façon sur le lien décisif entre phénoménalisation et écriture :

Un langage sans Leiblichkeit, i.e. un langage qui s'efface devant ce qu'il signifie, [devant] ce qu'il re-présente et donne à voir, est un langage qui transcrit, qui ne phénoménalise pas, c'est-à-dire qui ne présente pas, mais précisément qui représente quelque chose qui est déjà présent, i.e. déjà phénoménalisé. [...]

Pour que le langage phénoménalise, il ne doit avoir rien à dire, il ne doit rien exprimer. C'est l'écriture, au sens que Maurice Blanchot donne à ce mot, ou encore R. Barthes (l'écrire intransitif: l'écrire qui n'écrit rien). Pour ainsi dire, un langage qui s'engagerait sans vouloir dire quelque chose qui lui est extérieur, serait condamné à phénoménaliser. On ne peut donc dissocier pensée de la phénoménalisation et langage de la phénoménalisation. La phénoménalisation ne s'exprime pas dans un langage, la phénoménalisation s'écrit - au sens que lui donne Derrida (archi-écriture) - la phénoménalisation est diction.

Cette deuxième perspective - qui met donc en avant un mode du phénoménaliser différent de toute forme d'expression sur ou à propos de l'étant - n'est pas le dernier mot de Richir à propos de l'élimination d'une approche ontologique en phénoménologie - tant s'en faut. Il y a aussi un mode du phénoménaliser susceptible d'exprimer une logique interne, une cohérence, dans la processualité du sens se faisant, en général, et dans l'œuvre de Richir, en particulier, qui, toutefois, ne 
dévoile pas un nouveau contenu conceptuel (c'est en cela que consiste cette proximité avec la perspective éliminant l'ontologie). Aussi convient-il à présent de faire un pas au-delà et d'intégrer, dans ces analyses, certains développements tardifs de Richir, en nous demandant quels sont le rôle et la fonction de l'architectonique dans sa méthodologie.

\section{Phénoménalisation et architectonique}

Au fond, l'acception richirienne de l'architectonique n'a que peu de choses à voir avec celle de Kant, même si, bien entendu, elle en provient. Tout d'abord, Richir est loin de revendiquer un caractère systématique de la phénoménologie ( $c f$. l'architectonique comme art des systèmes), et surtout, il ne partage pas l'aspiration scientifique de Kant (rappelons que Kant définissait l'architectonique aussi comme la « doctrine [de ce qui relève] du scientifique (Lehre des Scientifischen)»). Néanmoins, il s'y réfère et ce, à travers son ancien doctorant Frank Pierobon.

Richir s'est toujours exprimé avec beaucoup de respect sur les travaux de ce dernier sur Kant, et il est évident que l'usage qu'il en a fait lui doit beaucoup. Mais comme il l'a lui-même dit dans ses conversations avec Sacha Carlson dans L'écart et le rien, même s'il a pris beaucoup d'intérêt à l'idée d'une "'organisation' a prio$r i »$ de l'esprit humain par une architectonique, il ne voulait ni ne pouvait adhérer au fantasme plus général, voire extrapolé, " d'une architectonique qui existerait a priori» (ER, p. 192). Qu' est-ce qui le gênait, au fond, dans cette conception-là de l'architectonique ? La fidélité à sa propre pensée du mouvement. L'architectonique au sens richirien ne saurait être statique, elle est mobile et dynamique. Mais qu' estce qui est exactement mobile et dynamique là-dedans? Nous le comprenons en précisant les termes de tectonique et d'archè. Richir entend par «tectonique » un ensemble de mouvements - qui sont autant de " recouvrements " que de "renversements » ou de " retournements» - des registres architectoniques qui ne sont pas des « couches » (il ne s'agit point là de «stratigraphie»), mais mettent en jeu des « archai », c'est-à-dire, au fond, des complexions notionnelles et conceptuelles qui font « tenir » cet « art [et non la science !] des systèmes ».

Cependant, il ne faut pas s'arrêter là. Cette compréhension de l'architectonique a aussi une répercussion sur le mouvement de l'œuvre de Richir, sur ses systèmes de coordonnées fondamentales - et donc sur les champs élémentaires de sa refonte de la phénoménologie. En général, on distingue parmi ces champs d'intérêt élémentaires de Richir ce qui relève du symbolique (de l'institution symbolique) et ce qui se réfère au phénoménologique. Or, à notre avis, et c'est cette nouvelle 
hypothèse que nous proposons de soumettre à la discussion, la notion d'architectonique peut aussi servir à délimiter de façon judicieuse une période importante de la production philosophique de Richir - la dernière qui s'est amorcée avec les Fragments phénoménologiques sur le langage de 2008, dans laquelle le mouvement de pensée de Richir se présente encore sous un autre jour. Le lecteur constate en effet que le style de Richir s'est énormément libéré dans ce livre (ainsi que dans les cinq autres qui vont encore suivre [La contingence du despote, Variations sur le sublime et le soi, Sur le sublime et le soi-Variations II, De la négativité en phénoménologie et Propositions buissonnières]) : il abandonne les explications détaillées de textes, il se livre à des développements totalement personnels, et même dans les titres, on constate une sorte de dissolution - de fragments, à variations pour finir avec des propositions. Et un point est ici absolument décisif : Richir ne procède plus à de nouvelles analyses (ce qui n'exclut pas l'introduction de concepts inédits), mais il laisse littéralement le sens se faire de manière à ce qu'il déploie ses conséquences sous-jacentes. Cela inclut en particulier l'idée que l'architectonique permet d'éviter l'aporie d'un " langage sans Leiblichkeit " qui s'effacerait devant ce qu'il signifie. ${ }^{12}$ Et ce qui est fort intéressant là-dedans, c' est que la mise en place de cette cohérence (de ce qui a été pensé de façon tacite) et de cette aspérité de l'écriture (à l'encontre d'une écriture lisse servant exclusivement à la communication du pensé) a un sens proprement phénoménologique et ne se limite nullement à une sorte de déduction à partir de ce qui a été posé antérieurement. On pourrait même aller jusqu'à dire - telle et donc notre hypothèse - que l'architectonique constitue un mode original et inédit de la phénoménalisation.

Dès lors, l'architectonique se présente aussi comme un moment très important du mouvement de pensée de Richir dans son ensemble : elle commande la cohérence ultime ou, plus exactement, elle donne lieu à des enchaînements et aussi, par moments, à des connexions entre des concepts, ce qui exige justement, par moment, de procéder à l'introduction de nouveaux concepts (qui consistent dans une explicitation [non une déduction, nous insistons] de ce qui a été mis en place auparavant) - on peut citer par exemple, à ce propos, l' " élément de l'intelligible » ou le "sublime négatif ». L'architectonique constitue ainsi non seulement la cohérence interne des élaborations richiriennes, mais elle désigne également un moment fondamental dans l'œuvre publiée de Richir en ouvrant un troisième champ à côté de ce qui est sous le signe du symboliquement institué et du phénoménologiquement dévoilé.

12 Nous remercions Jean-François Perrier de cette remarque tout à fait pertinente. 
Concluons. D’une manière générale, la problématique fondamentale de la refonte richirienne de la phénoménologie transcendantale concerne le rapport de l'apparaître, ou encore du phénomène et de sa phénoménalisation, à l'être. Dans l'œuvre richirienne, on peut mettre en évidence deux tendances à ce propos, qui souvent se déploient en parallèle. La première tendance fait part d'une profonde dissociation entre le paraître et l'être, elle défend la thèse d'une sorte d'élimination de l'ontologie en phénoménologie. La seconde tendance, au contraire, affirme qu'il est possible - et même nécessaire ! - de rendre compte de l'être dans et à travers la phénoménalisation. La présente étude s'est pleinement inscrite dans la première tendance. ${ }^{13}$ Elle a mis en évidence trois perspectives possibles. Premièrement, elle a montré que pour Richir toute assomption d'être, en phénoménologie, relève du simulacre. Deuxièmement, elle a mis en avant l'idée que le phénoménaliser ne doit pas être conçu comme un exprimer (sur ou à propos de quelque chose), mais relève d'une autre forme du faire apparaître (par exemple de l'écriture). Enfin, troisièmement, elle a fait voir en quoi l'architectonique peut présenter une forme de phénoménalisation qui, là encore, ne vise pas un étant objectif, mais une cohérence interne du sens (et d'une œuvre) en train de se faire. Or, ces deux perspectives fondamentales - l'une qui élimine, l'autre qui promeut une certaine acception de l'être - ne sont peut-être pas inconciliables. C'est ce qui a été pointé très judicieusement par Patrice Loraux à travers son idée de l' "irréductible » ${ }^{14}$, laquelle reprend autrement une conception développée plus haut - à savoir le zigzag entre deux acceptions de la positivité, celle au-delà de la fluidité de l'apparence (ou de la phénoménalité) et celle, la plus enfouie (donc dans un en-deçà), qui fixe cette fluidité, mais non pas pour la pétrifier, mais pour rendre possible son accès et sa schématisation. Ultimement, ce rapport fait d'ailleurs également écho à la conception richirienne du Soi qui se tient à son tour dans la tension entre une fluidification et une fixation nécessaire, pour que le procès du sens se faisant puisse se déployer de façon rigoureuse en régime phénoménologique.

Alexander Schnell est professeur de philosophie à l>Université de Wuppertal. Il y dirige l'Institut für Transzendentalphilosophie und Phänomenologie (ITP) et les Archives Marc Richir (MRA). Publications (séléction): En deçà du sujet. Du

13 Pour la seconde, et en particulier en ce qui concerne le « double mouvement de la phénoménalisation » dans "Le rien enroulé » (paru en 1970) de M. Richir, cf. notre article " Marc Richir et la métaphysique ", in Annales de Phénoménologie - nouvelle série ", n 18/2019.

14 Voir Loraux Patrice, "Pour n'en pas finir», in Annales de phénoménologie, n 15/2016, pp. 7-14. $C f$. aussi, sur ce point, notre étude " Réflexions sur le ,mouvement de pensée de Marc Richir », in : Arrien, S. -J., Perrier, J.-F. et Hardy, J.-S. (Ed.), Aux marges de la phénoménologie. Lectures de Marc Richir, Paris, Hermann, coll. « Rue de la Sorbonne », 2019. 
temps dans la philosophie transcendantale allemande, "Epiméthée », Paris, PUF, 2010; Hinaus. Entwürfe zu einer phänomenologischen Metaphysik und Anthropologie, Würzburg, Königshausen \& Neumann, Orbis Phaenomenologicus (Studien), $\mathrm{n}^{\circ}$ 24, 2011; Le sens se faisant. Marc Richir et la refondation de la phénoménologie transcendantale, Brüssel, Ousia, 2011; En voie du réel, Paris, Hermann, "Le Bel Aujourd'hui”, 2013; Qu'est-ce que le phénomène?, Paris, Vrin, 2014; La déhiscence du sens, Paris, Hermann, "Le Bel Aujourd'hui", 2015; Lire les Beiträge zur Philosophie de Heidegger, A. Schnell (éd.), Paris, Hermann, 2017; Qu'est-ce que la phénoménologie transcendentale?, Grenoble, Millon, 2020. 